\title{
PERMANENCE FOR A CLASS OF NONLINEAR \\ DIFFERENCE SYSTEMS
}

BINXIANG DAI AND XINGFU ZOU

Received 22 January 2006; Accepted 13 March 2006

A class of nonlinear difference systems is considered in this paper. By exploring the relationship between this system and a correspondent first-order difference system, some permanence results are obtained.

Copyright (c) 2006 B. Dai and X. Zou. This is an open access article distributed under the Creative Commons Attribution License, which permits unrestricted use, distribution, and reproduction in any medium, provided the original work is properly cited.

\section{Introduction}

Consider the following system of nonlinear difference equations:

$$
x_{n+1}=\lambda x_{n}+f\left(\alpha_{1} y_{n}-\beta_{1} y_{n-1}\right), \quad y_{n+1}=\lambda y_{n}+f\left(\alpha_{2} x_{n}-\beta_{2} x_{n-1}\right),
$$

where $\lambda \in(0,1), \alpha_{i}, \beta_{i}(i=1,2)$ are given positive constants, and $f: \mathbb{R} \rightarrow \mathbb{R}$ is a real function. System (1.1) can be regarded as the discrete analog of the following neural network of two neurons with dynamical threshold effects:

$$
\begin{aligned}
& \frac{d x(t)}{d t}=-\mu x(t)+f\left(\alpha_{1} y(t)-\beta_{1} y(t-\tau)\right), \\
& \frac{d y(t)}{d t}=-\mu y(t)+f\left(\alpha_{2} x(t)-\beta_{2} x(t-\tau)\right) .
\end{aligned}
$$

System (1.2) has found interesting applications in, for example, temporal evolution of sublattice magnetization (see [3]). Recently, the dynamics of (1.2) and some related models have been discussed in $[1,2,5]$.

System (1.1) can also be viewed as an extension to two dimensions of the equation

$$
x_{n+1}=\lambda x_{n}+f\left(x_{n}-x_{n-1}\right),
$$

Hindawi Publishing Corporation

Discrete Dynamics in Nature and Society

Volume 2006, Article ID 78607, Pages 1-10

DOI 10.1155/DDNS/2006/78607 


\section{Permanence for a class of nonlinear difference systems}

which has been studied by Sedaghat [6] and other authors (see [4, 7]). By exploring the relationship between (1.3) and the following first-order initial value problem:

$$
v_{n+1}=f\left(v_{n}\right), \quad v_{1}=x_{1}-x_{0},
$$

some sufficient conditions for the permanence of (1.3) are obtained in [6]. It is natural to expect that similar results in [6] can be extended from (1.3) to system (1.1). This is the goal of this paper.

As usual, system (1.1) is said to be permanent, if there exists a compact set $\Omega$ in the interior of $\mathbb{R} \times \mathbb{R}$ such that any solution of (1.1) will ultimately stay in $\Omega$.

The organization of this paper is as follows. In Section 2, we discuss the following difference system:

$$
u_{n+1}=f\left(\alpha_{1} v_{n}\right), \quad v_{n+1}=f\left(\alpha_{2} u_{n}\right), \quad n=1,2, \ldots,
$$

and give some propositions which address the permanence of system (1.5), and therefore which themselves are of some interest and importance. In Section 3, by setting up a useful relationship between systems (1.1) and (1.5), we obtain some sufficient conditions for the permanence of system (1.1). An important example is given in Section 4 .

\section{Basic propositions}

In this section, we discuss some properties of system (1.5). For convenience, we will adopt some notations as follows:

$$
g:=\alpha_{1} f, \quad h:=\alpha_{2} f, \quad F^{2}:=F \circ F, \quad F^{n}:=F \circ F^{n-1}, \quad n=2,3, \ldots,
$$

where $F \circ G(x)=F(G(x))$.

It is easy to have the following proposition.

Proposition 2.1. Every solution $\left\{\left(u_{n}, v_{n}\right)\right\}_{n \in \mathbb{N}}$ of system (1.5) satisfies

$$
\begin{aligned}
& u_{n+1}= \begin{cases}f \circ(g \circ h)^{k-1} \circ g\left(\alpha_{2} u_{1}\right), & \text { if } n=2 k, \\
f \circ(g \circ h)^{k}\left(\alpha_{1} v_{1}\right), & \text { if } n=2 k+1,\end{cases} \\
& v_{n+1}= \begin{cases}f \circ(h \circ g)^{k-1} \circ g\left(\alpha_{1} v_{1}\right), & \text { if } n=2 k, \\
f \circ(h \circ g)^{k}\left(\alpha_{2} u_{1}\right), & \text { if } n=2 k+1 .\end{cases}
\end{aligned}
$$

Proposition 2.2. Let $f: \mathbb{R} \rightarrow \mathbb{R}$ be a nondecreasing function. Assume that the following condition holds.

$\left(\mathrm{H}_{1}\right)$ There exist $\delta_{i} \in(0,1)$ and $M_{1}>0$ such that for all $x \geq M_{1}$,

$$
f\left(\alpha_{i} x\right) \leq \delta_{i} x, \quad i=1,2 .
$$

Then every solution of (1.5) is eventually bounded from above (independent of initial conditions). 
Proof. Let $\left\{\left(u_{n}, v_{n}\right)\right\}$ be a solution of (1.5). We claim that there exists a positive integer $m$ such that

$$
u_{m}<M_{1}, \quad v_{m}<M_{1} \text {. }
$$

First we can prove that there is an $m_{1}$ such that $u_{m_{1}}<M_{1}$. Otherwise, for any $n>0$, we have $u_{n} \geq M_{1}$. Then

$$
\begin{aligned}
& v_{n+1}=f\left(\alpha_{2} u_{n}\right) \leq \delta_{2} u_{n}<u_{n}, \\
& u_{n+2}=f\left(\alpha_{1} v_{n+1}\right) \leq f\left(\alpha_{1} u_{n}\right) \leq \delta_{1} u_{n}, \\
& v_{n+3}=f\left(\alpha_{2} u_{n+2}\right) \leq \delta_{2} u_{n+2}<u_{n+2}, \\
& u_{n+4}=f\left(\alpha_{1} v_{n+3}\right) \leq f\left(\alpha_{1} u_{n+2}\right) \leq \delta_{1} u_{n+2} \leq \delta_{1}^{2} u_{n} .
\end{aligned}
$$

It follows, by induction, that

$$
u_{n+2 k} \leq \delta_{1}^{k} u_{n}, \quad k=1,2, \ldots
$$

Now, fix $n$ and take $k \rightarrow \infty$ in (2.6) and note that $0<\delta_{1}<1$, we then get

$$
\lim _{k \rightarrow \infty} u_{n+2 k}=0
$$

which contradicts to $u_{n} \geq M_{1}>0$.

Next we distinguish two cases.

Case 1. If $v_{m_{1}}<M_{1}$, then (2.4) holds.

Case 2. If $v_{m_{1}} \geq M_{1}$, we show that there exists $k_{1}$ such that

$$
v_{m_{1}+2 k_{1}}<M_{1} .
$$

Assume that (2.8) is not true, then $v_{m_{1}+2 k} \geq M_{1}$ for all $k$. Similar to the proof of (2.6), we have

$$
0<M_{1} \leq v_{m_{1}+2 k} \leq \delta_{2}^{k} v_{m_{1}} \longrightarrow 0 \quad(\text { as } k \longrightarrow \infty)
$$

which is contradiction.

Noting $u_{m_{1}}<M_{1}$ implies that $u_{m_{1}+2 k}<M_{1}$ for all $k$, then take $m=m_{1}+2 k_{1}$, and (2.4) holds.

Now, by (1.5), we have

$$
\begin{aligned}
& u_{m+1}=f\left(\alpha_{1} v_{m}\right) \leq f\left(\alpha_{1} M_{1}\right) \leq \delta_{1} M_{1}<M_{1}, \\
& v_{m+1}=f\left(\alpha_{2} u_{m}\right) \leq f\left(\alpha_{2} M_{1}\right) \leq \delta_{2} M_{1}<M_{1} .
\end{aligned}
$$

Thus, by induction, we obtain

$$
u_{n}<M_{1}, \quad v_{n}<M_{1}
$$

for all $n \geq m$. This completes the proof. 
4 Permanence for a class of nonlinear difference systems

Letting $u_{n}^{\prime}=-u_{n}, v_{n}^{\prime}=-v_{n}, F(x)=-f(-x)$, we then have the following proposition which comes directly from Proposition 2.2.

Proposition 2.3. Let $f: \mathbb{R} \rightarrow \mathbb{R}$ be a nondecreasing function. Assume that the following condition holds.

$\left(\mathrm{H}_{2}\right)$ There exist $\delta_{i} \in(0,1)$ and $M_{2}>0$ such that for all $x \leq-M_{2}$,

$$
f\left(\alpha_{i} x\right) \geq \delta_{i} x, \quad i=1,2 .
$$

Then every solution of (1.5) is eventually bounded from below (independent of initial conditions).

Propositions 2.2 and 2.3 can be combined to give the following proposition.

Proposition 2.4. Let $f: \mathbb{R} \rightarrow \mathbb{R}$ be a nondecreasing function. If there exist $\delta_{i} \in(0,1)$ such that

$$
\lim _{x \rightarrow \infty} \frac{f\left(\alpha_{i} x\right)}{x}=\delta_{i}, \quad i=1,2,
$$

then (1.5) is permanent.

\section{Permanence of (1.1)}

In this section, we are concerned with the permanence of system (1.1). To this end, we need to establish the following lemma which gives a useful link between the solutions of (1.1) and (1.5).

Lemma 3.1. Suppose that $f: \mathbb{R} \rightarrow \mathbb{R}$ is a nondecreasing function. Let $\left\{\left(x_{n}, y_{n}\right)\right\}$ be a nonnegative solution of the following difference inequalities:

$$
x_{n+1} \leq \lambda x_{n}+f\left(\alpha_{1} y_{n}-\beta_{1} y_{n-1}\right), \quad y_{n+1} \leq \lambda y_{n}+f\left(\alpha_{2} x_{n}-\beta_{2} x_{n-1}\right),
$$

with initial conditions $\left(x_{0}, y_{0}\right)$ and $\left(x_{1}, y_{1}\right)$, and $\left\{\left(u_{n}, v_{n}\right)\right\}$ is the solution of $(1.5)$ with the initial values $u_{1}, v_{1}$ satisfying

$$
\alpha_{2} u_{1}=\alpha_{2} x_{1}-\beta_{2} x_{0}, \quad \alpha_{1} v_{1}=\alpha_{1} y_{1}-\beta_{1} y_{0}
$$

If the following condition holds:

$\left(\mathrm{H}_{3}\right) \alpha_{i} \lambda-\beta_{i} \leq 0, i=1,2$,

then for all $n \geq 1$,

$$
\alpha_{2} x_{n} \leq \lambda^{n-1} \beta_{2} x_{0}+\sum_{k=1}^{n} \lambda^{n-k} \alpha_{2} u_{k}, \quad \alpha_{1} y_{n} \leq \lambda^{n-1} \beta_{1} y_{0}+\sum_{k=1}^{n} \lambda^{n-k} \alpha_{1} v_{k} .
$$

Proof. We first observe that

$$
\alpha_{2} x_{1}=\beta_{2} x_{0}+\alpha_{2} u_{1}, \quad \alpha_{1} y_{1}=\beta_{1} y_{0}+\alpha_{1} v_{1}
$$


and that

$$
\begin{aligned}
\alpha_{2} x_{2} & \leq \alpha_{2}\left(\lambda x_{1}+f\left(\alpha_{1} y_{1}-\beta_{1} y_{0}\right)\right) \\
& =\lambda\left(\beta_{2} x_{0}+\alpha_{2} u_{1}\right)+\alpha_{2} f\left(\alpha_{1} v_{1}\right)=\lambda \beta_{2} x_{0}+\lambda \alpha_{2} u_{1}+\alpha_{2} u_{2}, \\
\alpha_{1} y_{2} & \leq \alpha_{1}\left(\lambda y_{1}+f\left(\alpha_{2} x_{1}-\beta_{2} x_{0}\right)\right) \\
& =\lambda\left(\beta_{1} y_{0}+\alpha_{1} v_{1}\right)+\alpha_{1} f\left(\alpha_{2} u_{1}\right)=\lambda \beta_{1} y_{0}+\lambda \alpha_{1} v_{1}+\alpha_{1} v_{2} .
\end{aligned}
$$

Hence, (3.3) holds for $n=1,2$. Next we assume that (3.3) holds for all integers less than or equal to some integer $n$. Then

$$
\begin{aligned}
\alpha_{2} x_{n+1} & \leq \alpha_{2}\left(\lambda x_{n}+f\left(\alpha_{1} y_{n}-\beta_{1} y_{n-1}\right)\right) \\
& \leq \lambda^{n} \beta_{2} x_{0}+\sum_{k=1}^{n} \lambda^{n-k+1} \alpha_{2} u_{k}+\alpha_{2} f\left(\alpha_{1} y_{n}-\beta_{1} y_{n-1}\right), \\
\alpha_{1} y_{n+1} & \leq \alpha_{1}\left(\lambda y_{n}+f\left(\alpha_{2} y_{n}-\beta_{2} x_{n-1}\right)\right) \\
& \leq \lambda^{n} \beta_{1} y_{0}+\sum_{k=1}^{n} \lambda^{n-k+1} \alpha_{1} v_{k}+\alpha_{1} f\left(\alpha_{2} x_{n}-\beta_{2} x_{n-1}\right) .
\end{aligned}
$$

So it remains to show that

$$
f\left(\alpha_{1} y_{n}-\beta_{1} y_{n-1}\right) \leq u_{n+1}, \quad f\left(\alpha_{2} x_{n}-\beta_{2} x_{n-1}\right) \leq v_{n+1} .
$$

To this end, we note that

$$
\begin{aligned}
\alpha_{1} y_{n}-\beta_{1} y_{n-1} & \leq\left(\alpha_{1} \lambda-\beta_{1}\right) y_{n-1}+\alpha_{1} f\left(\alpha_{2} x_{n-1}-\beta_{2} x_{n-2}\right) \\
& \leq \alpha_{1} f\left(\alpha_{2} x_{n-1}-\beta_{2} x_{n-2}\right)=g\left(\alpha_{2} x_{n-1}-\beta_{2} x_{n-2}\right), \\
\alpha_{2} x_{n}-\beta_{2} x_{n-1} & \leq\left(\alpha_{2} \lambda-\beta_{2}\right) x_{n-1}+\alpha_{2} f\left(\alpha_{1} y_{n-1}-\beta_{1} y_{n-2}\right) \\
& \leq \alpha_{2} f\left(\alpha_{1} y_{n-1}-\beta_{1} y_{n-2}\right)=h\left(\alpha_{1} y_{n-1}-\beta_{1} y_{n-2}\right),
\end{aligned}
$$

which, together with the assumption that $f$ is nondecreasing, implies that

$$
\begin{aligned}
& f\left(\alpha_{1} y_{n}-\beta_{1} y_{n-1}\right) \leq f \circ g\left(\alpha_{2} x_{n-1}-\beta_{2} x_{n-2}\right), \\
& f\left(\alpha_{2} x_{n}-\beta_{2} x_{n-1}\right) \leq f \circ h\left(\alpha_{1} y_{n-1}-\beta_{1} y_{n-2}\right) .
\end{aligned}
$$

Following this fashion, we can get

$$
\begin{aligned}
& f\left(\alpha_{1} y_{n}-\beta_{1} y_{n-1}\right) \leq \begin{cases}f \circ(g \circ h)^{k-1} \circ g\left(\alpha_{2} u_{1}\right), & \text { if } n=2 k, \\
f \circ(g \circ h)^{k}\left(\alpha_{1} v_{1}\right), & \text { if } n=2 k+1,\end{cases} \\
& f\left(\alpha_{2} x_{n}-\beta_{2} x_{n-1}\right) \leq \begin{cases}f \circ(h \circ g)^{k-1} \circ g\left(\alpha_{1} v_{1}\right), & \text { if } n=2 k, \\
f \circ(h \circ g)^{k}\left(\alpha_{2} u_{1}\right), & \text { if } n=2 k+1 .\end{cases}
\end{aligned}
$$

Then (3.7) follows from Proposition 2.1 and thus the proof is complete. 
6 Permanence for a class of nonlinear difference systems

Similar to the proof of Lemma 3.1, we have the following.

Lemma 3.2. Suppose that $f: \mathbb{R} \rightarrow \mathbb{R}$ is a nondecreasing function. Let $\left\{\left(x_{n}, y_{n}\right)\right\}$ be a nonpositive solution of the following difference inequalities:

$$
x_{n+1} \geq \lambda x_{n}+f\left(\alpha_{1} y_{n}-\beta_{1} y_{n-1}\right), \quad y_{n+1} \geq \lambda y_{n}+f\left(\alpha_{2} x_{n}-\beta_{2} x_{n-1}\right),
$$

with initial conditions $\left(x_{0}, y_{0}\right)$ and $\left(x_{1}, y_{1}\right)$, and $\left\{\left(u_{n}, v_{n}\right)\right\}$ is the solution of (1.5) with the initial values $u_{1}, v_{1}$ satisfying (3.2). If the condition $\left(H_{3}\right)$ holds, then for all $n \geq 1$,

$$
\alpha_{2} x_{n} \geq \lambda^{n-1} \beta_{2} x_{0}+\sum_{k=1}^{n} \lambda^{n-k} \alpha_{2} u_{k}, \alpha_{1} y_{n} \geq \lambda^{n-1} \beta_{1} y_{0}+\sum_{k=1}^{n} \lambda^{n-k} \alpha_{1} v_{k} .
$$

We are now able to state and prove our permanence results for system (1.1).

Theorem 3.3. Let $f$ be nondecreasing and bounded from below on $\mathbb{R}$. Suppose that $\left(H_{1}\right)$ and $\left(\mathrm{H}_{3}\right)$ hold. Assume further that

$\left(\mathrm{H}_{4}\right) \alpha_{i} \geq \beta_{i}, i=1,2$.

Then (1.1) is permanent.

Proof. If we define $X_{n}=f\left(\alpha_{2} x_{n}-\beta_{2} x_{n-1}\right), Y_{n}=f\left(\alpha_{1} y_{n}-\beta_{1} y_{n-1}\right)$ for all $n \geq 1$, then it follows inductively from (1.1) that

$$
x_{n}=\lambda^{n-1} x_{1}+\sum_{k=1}^{n-1} \lambda^{n-k-1} Y_{k}, \quad y_{n}=\lambda^{n-1} y_{1}+\sum_{k=1}^{n-1} \lambda^{n-k-1} X_{k} .
$$

Let $L_{0}$ be a lower bound for $f(t)$ and without loss of generality we assume that $L_{0} \leq 0$. As $X_{k} \geq L_{0}$ and $Y_{k} \geq L_{0}$ for all $k$, we conclude from (3.13) that for all $n$,

$$
x_{n} \geq \lambda^{n-1} x_{1}+\frac{\left(1-\lambda^{n-1}\right) L_{0}}{1-\lambda}, \quad y_{n} \geq \lambda^{n-1} y_{1}+\frac{\left(1-\lambda^{n-1}\right) L_{0}}{1-\lambda},
$$

and therefore $\left\{\left(x_{n}, y_{n}\right)\right\}$ is bounded from below. In fact, it is clear that there is a positive integer $n_{0}$ such that for all $n \geq n_{0}$,

$$
x_{n} \geq L, \quad y_{n} \geq L,
$$

where $L=L_{0} /(1-\lambda)-1<0$. We next show that $\left\{\left(x_{n}, y_{n}\right)\right\}$ is bounded from above as well. Define

$$
\phi_{n}=x_{n+n_{0}}-L, \quad \varphi_{n}=y_{n+n_{0}}-L
$$

for all $n \geq 0$, so that $\phi_{n} \geq 0, \varphi_{n} \geq 0$ for all $n$. Now for each $n \geq 1$, we have

$$
\begin{aligned}
& \phi_{n+1}=\lambda x_{n+n_{0}}+f\left(\alpha_{1} y_{n+n_{0}}-\beta_{1} y_{n+n_{0}-1}\right)-L=\lambda \phi_{n}+f\left(\alpha_{1} y_{n+n_{0}}-\beta_{1} y_{n+n_{0}-1}\right)-(1-\lambda) L, \\
& \varphi_{n+1}=\lambda y_{n+n_{0}}+f\left(\alpha_{2} x_{n+n_{0}}-\beta_{2} x_{n+n_{0}-1}\right)-L=\lambda \varphi_{n}+f\left(\alpha_{2} x_{n+n_{0}}-\beta_{2} x_{n+n_{0}-1}\right)-(1-\lambda) L .
\end{aligned}
$$


Note that

$$
\begin{gathered}
\alpha_{1} y_{n+n_{0}}-\beta_{1} y_{n+n_{0}-1}=\alpha_{1} \varphi_{n}-\beta_{1} \varphi_{n-1}+\left(\alpha_{1}-\beta_{1}\right) L \leq \alpha_{1} \varphi_{n}-\beta_{1} \varphi_{n-1}, \\
\alpha_{2} x_{n+n_{0}}-\beta_{2} x_{n+n_{0}-1}=\alpha_{2} \phi_{n}-\beta_{2} \phi_{n-1}+\left(\alpha_{2}-\beta_{2}\right) L \leq \alpha_{2} \phi_{n}-\beta_{2} \phi_{n-1},
\end{gathered}
$$

which, together with the assumption that $f$ is nondecreasing, implies that

$$
\begin{aligned}
& f\left(\alpha_{1} y_{n+n_{0}}-\beta_{1} y_{n+n_{0}-1}\right) \leq f\left(\alpha_{1} \varphi_{n}-\beta_{1} \varphi_{n-1}\right), \\
& f\left(\alpha_{2} x_{n+n_{0}}-\beta_{2} x_{n+n_{0}-1}\right) \leq f\left(\alpha_{2} \phi_{n}-\beta_{2} \phi_{n-1}\right) .
\end{aligned}
$$

Define $F(x):=f(x)-(1-\lambda) L$. By (3.17) and (3.19), we get

$$
\phi_{n+1} \leq \lambda \phi_{n}+F\left(\alpha_{1} \varphi_{n}-\beta_{1} \varphi_{n-1}\right), \quad \varphi_{n+1} \leq \lambda \varphi_{n}+F\left(\alpha_{2} \phi_{n}-\beta_{2} \phi_{n-1}\right) .
$$

Let $\delta_{i}^{*} \in\left(\delta_{i}, 1\right), i=1,2$, and $M_{1}^{*}=\max \left\{M_{1},-(1-\lambda) L /\left(\delta_{1}^{*}-\delta_{1}\right),-(1-\lambda) L /\left(\delta_{2}^{*}-\delta_{2}\right)\right\}$. It is readily verified that for all $x \geq M_{1}^{*}$,

$$
F\left(\alpha_{i} x\right) \leq \delta_{i}^{*} x \quad(i=1,2) .
$$

Consider the following initial value problem:

$$
\begin{array}{ll}
u_{n+1}=F\left(\alpha_{1} v_{n}\right), & u_{1}=\frac{\alpha_{2} \phi_{1}-\beta_{2} \phi_{0}}{\alpha_{2}}, \\
v_{n+1}=F\left(\alpha_{2} u_{n}\right), & v_{1}=\frac{\alpha_{1} \varphi_{1}-\beta_{1} \varphi_{0}}{\alpha_{1}} .
\end{array}
$$

From Proposition 2.2 we know that there exist integer $m \geq 0$ and constant $M_{0}>0$ such that for all $n \geq m, u_{n} \leq M_{0}, v_{n} \leq M_{0}$. Applying Lemma 3.1 to (3.20), we obtain that for all $n \geq m$,

$$
\begin{aligned}
\alpha_{2} \phi_{n} & \leq \lambda^{n-1} \beta_{2} \phi_{0}+\sum_{k=1}^{m-1} \lambda^{n-k} \alpha_{2} u_{k}+\sum_{k=m}^{n} \lambda^{n-k} \alpha_{2} u_{k} \\
& \leq \lambda^{n-m+1}\left(\lambda^{m-2} \beta_{2} \phi_{0}+\lambda^{m-2} \alpha_{2} u_{1}+\cdots+\alpha_{2} u_{m-1}\right)+\alpha_{2} M_{0} \sum_{k=0}^{n-m} \lambda^{k} \\
& =\lambda^{n-m+1} M^{*}+\alpha_{2} M_{0}(1-\lambda)^{-1}\left(1-\lambda^{n-m+1}\right), \\
\alpha_{1} \varphi_{n} & \leq \lambda^{n-1} \beta_{1} \varphi_{0}+\sum_{k=1}^{m-1} \lambda^{n-k} \alpha_{1} v_{k}+\sum_{k=m}^{n} \lambda^{n-k} \alpha_{1} v_{k} \\
& \leq \lambda^{n-m+1}\left(\lambda^{m-2} \beta_{1} \varphi_{0}+\lambda^{m-2} \alpha_{1} v_{1}+\cdots+\alpha_{1} v_{m-1}\right)+\alpha_{1} M_{0} \sum_{k=0}^{n-m} \lambda^{k} \\
& =\lambda^{n-m+1} N^{*}+\alpha_{1} M_{0}(1-\lambda)^{-1}\left(1-\lambda^{n-m+1}\right),
\end{aligned}
$$


8 Permanence for a class of nonlinear difference systems

where $M^{*}=\lambda^{m-2} \beta_{2} \phi_{0}+\lambda^{m-2} \alpha_{2} u_{1}+\cdots+\alpha_{2} u_{m-1}, N^{*}=\lambda^{m-2} \beta_{1} \varphi_{0}+\lambda^{m-2} \alpha_{1} v_{1}+\cdots+$ $\alpha_{1} v_{m-1}$. Thus there exists $n_{1} \geq m$ such that for all $n \geq n_{1}$,

$$
\phi_{n} \leq \frac{M_{0}}{1-\lambda}+1, \quad \varphi_{n} \leq \frac{M_{0}}{1-\lambda}+1
$$

Hence, for all $n \geq n_{0}+n_{1}$, we have

$$
\left(x_{n}, y_{n}\right) \in[L, M] \times[L, M]
$$

where

$$
M=\frac{M_{0}}{1-\lambda}+1+L
$$

This shows that (1.1) is permanent. The proof is completed.

Similarly, we have the following.

TheOREM 3.4. Let $f$ be nondecreasing and bounded from above on $\mathbb{R}$. Suppose that $\left(\mathrm{H}_{2}\right)$, $\left(H_{3}\right)$, and $\left(H_{4}\right)$ hold. Then (1.1) is permanent.

From the proof of Theorem 3.3, we can easily establish the following assertion.

COROLlary 3.5. Let $f$ be bounded from below (from above) on $\mathbb{R}$. Then every solution of (1.1) is bounded from below (from above). In particular, if $f$ is bounded, then every solution of (1.1) is bounded.

\section{An example}

Consider the following system of two difference equations:

$$
X_{n+1}=\lambda X_{n}+\alpha_{1} f\left(Y_{n}\right)-\beta_{1} f\left(Y_{n-1}\right), \quad Y_{n+1}=\lambda Y_{n}+\alpha_{2} f\left(X_{n}\right)-\beta_{2} f\left(X_{n-1}\right),
$$

where $\lambda \in[0,1), \alpha_{i}, \beta_{i}(i=1,2)$ are given positive constants with, and $f: \mathbb{R} \rightarrow \mathbb{R}$ is a real function.

Let $\left\{\left(X_{n}, Y_{n}\right)\right\}$ be a solution of (4.1), and for $n \geq 1$, define

$$
\begin{aligned}
& x_{n}=\left(\frac{\beta_{2}}{\alpha_{2}}\right)^{n} x_{0}+\sum_{k=0}^{n-1}\left(\frac{\beta_{2}}{\alpha_{2}}\right)^{n-k-1} \frac{1}{\alpha_{2}} Y_{k} \\
& y_{n}=\left(\frac{\beta_{1}}{\alpha_{1}}\right)^{n} y_{0}+\sum_{k=0}^{n-1}\left(\frac{\beta_{1}}{\alpha_{1}}\right)^{n-k-1} \frac{1}{\alpha_{1}} X_{k},
\end{aligned}
$$

for some real numbers $x_{0}, y_{0}$. We will show that $\left\{\left(x_{n}, y_{n}\right)\right\}$ satisfies (1.1) for some choice 
of $\left(x_{0}, y_{0}\right)$. Note that

$$
\begin{gathered}
X_{n}=\alpha_{1} y_{n+1}-\beta_{1} y_{n}, \quad Y_{n}=\alpha_{2} x_{n+1}-\beta_{2} x_{n}, \\
x_{2}=\left(\frac{\beta_{2}}{\alpha_{2}}\right)^{2} x_{0}+\frac{\beta_{2}}{\alpha_{2}^{2}} Y_{0}+\frac{1}{\alpha_{2}} Y_{1} \\
y_{2}=\left(\frac{\beta_{1}}{\alpha_{1}}\right)^{2} y_{0}+\frac{\beta_{1}}{\alpha_{1}^{2}} X_{0}+\frac{1}{\alpha_{1}} X_{1} .
\end{gathered}
$$

In order for $\left\{\left(x_{n}, y_{n}\right)\right\}$ to satisfy $(1.1), x_{0}$ and $y_{0}$ must be chosen such that

$$
\begin{aligned}
& \lambda x_{1}+f\left(\alpha_{1} y_{1}-\beta_{1} y_{0}\right)=\lambda\left(\frac{\beta_{2}}{\alpha_{2}} x_{0}+\frac{1}{\alpha_{2}} Y_{0}\right)+f\left(X_{0}\right), \\
& \lambda y_{1}+f\left(\alpha_{2} x_{1}-\beta_{2} x_{0}\right)=\lambda\left(\frac{\beta_{1}}{\alpha_{1}} y_{0}+\frac{1}{\alpha_{1}} X_{0}\right)+f\left(Y_{0}\right) .
\end{aligned}
$$

Solving for $x_{0}$ and $y_{0}$ we obtain

$$
\begin{aligned}
& x_{0}=-\frac{1}{\beta_{2}} Y_{0}-\frac{\alpha_{2}}{\beta_{2}\left(\beta_{2}-\lambda \alpha_{2}\right)} Y_{1}+\frac{\alpha_{2}^{2}}{\beta_{2}\left(\beta_{2}-\lambda \alpha_{2}\right)} f\left(X_{0}\right) \\
& y_{0}=-\frac{1}{\beta_{1}} X_{0}-\frac{\alpha_{1}}{\beta_{1}\left(\beta_{1}-\lambda \alpha_{1}\right)} X_{1}+\frac{\alpha_{1}^{1}}{\beta_{1}\left(\beta_{1}-\lambda \alpha_{1}\right)} f\left(Y_{0}\right)
\end{aligned}
$$

Thus,

$$
x_{2}=\lambda x_{1}+f\left(\alpha_{1} y_{1}-\beta_{1} y_{0}\right), \quad y_{2}=\lambda y_{1}+f\left(\alpha_{2} x_{1}-\beta_{2} x_{0}\right) .
$$

Now, for any $n \geq 1$, from (4.1) and (4.3), we have

$$
\begin{aligned}
& \alpha_{2}\left[x_{n+2}-\lambda x_{n+1}-f\left(\alpha_{1} y_{n+1}-\beta_{1} y_{n}\right)\right]=\beta_{2}\left[x_{n+1}-\lambda x_{n}-f\left(\alpha_{1} y_{n}-\beta_{1} y_{n-1}\right)\right] \\
& \alpha_{1}\left[y_{n+2}-\lambda y_{n+1}-f\left(\alpha_{2} x_{n+1}-\beta_{2} x_{n}\right)\right]=\beta_{1}\left[y_{n+1}-\lambda y_{n}-f\left(\alpha_{2} x_{n}-\beta_{2} x_{n-1}\right)\right] .
\end{aligned}
$$

By (4.7) and (4.8), we can get inductively that $\left\{\left(x_{n}, y_{n}\right)\right\}$ is the solution of (1.1). From (4.3), we know

$$
\left|X_{n}\right| \leq \alpha_{1}\left|y_{n+1}\right|+\beta_{1}\left|y_{n}\right|, \quad\left|Y_{n}\right| \leq \alpha_{2}\left|x_{n+1}\right|+\beta_{2}\left|x_{n}\right|
$$

Therefore, by Theorems 3.3 and 3.4, we obtain the following result on permanence in system (4.1).

Corollary 4.1. Let $f$ be nondecreasing and bounded from below (or from above) on $\mathbb{R}$. Suppose that conditions $\left(H_{1}\right)$ (or $\left.\left(H_{2}\right)\right),\left(H_{3}\right)$, and $\left(H_{4}\right)$ hold. Then system (4.1) is permanent. 


\section{Acknowledgment}

This research was partially supported by the National Natural Science Foundation of China (Grant no. 10471153) and Natural Science Foundation of Central South University.

\section{References}

[1] K. Gopalsamy and I. K. C. Leung, Delay induced periodicity in a neural netlet of excitation and inhibition, Physica D. Nonlinear Phenomena 89 (1996), no. 3-4, 395-426.

[2] _ Convergence under dynamical thresholds with delays, IEEE Transactions on Neural Networks 8 (1997), no. 2, 341-348.

[3] R. Kuhn and J. L. van Hemmen, Temporal Association: Models of Neural Networks, edited by E. Domany, J. L. van Hemmen, and K. Schulten, Physics of Neural Networks, Springer, Berlin, 1991.

[4] G. Papaschinopoulos and C. J. Schinas, Stability of a class of nonlinear difference equations, Journal of Mathematical Analysis and Applications 230 (1999), no. 1, 211-222.

[5] S. G. Ruan and J. Wei, Periodic solutions of planar systems with two delays, Proceedings of the Royal Society of Edinburgh. Section A. Mathematics 129 (1999), no. 5, 1017-1032.

[6] H. Sedaghat, A class of nonlinear second order difference equations from macroeconomics, Nonlinear Analysis. Theory, Methods \& Applications 29 (1997), no. 5, 593-603.

[7] __ Bounded oscillations in the Hicks business cycle model and other delay equations, Journal of Difference Equations and Applications 4 (1998), no. 4, 325-341.

Xingfu Zou: Department of Applied Mathematics, University of Western Ontario, London, ON, Canada N6A 5B7

E-mail address:xzou@uwo.ca

Binxiang Dai: School of Mathematical Science and Computing Technology,

Central South University, Changsha, Hunan 410075, China

E-mail address: bxdai@hnu.cn 


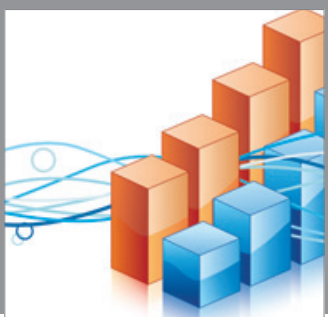

Advances in

Operations Research

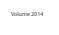

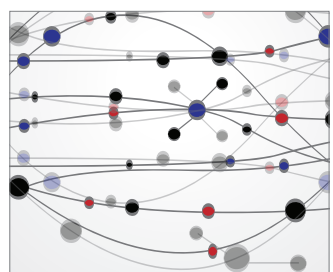

\section{The Scientific} World Journal
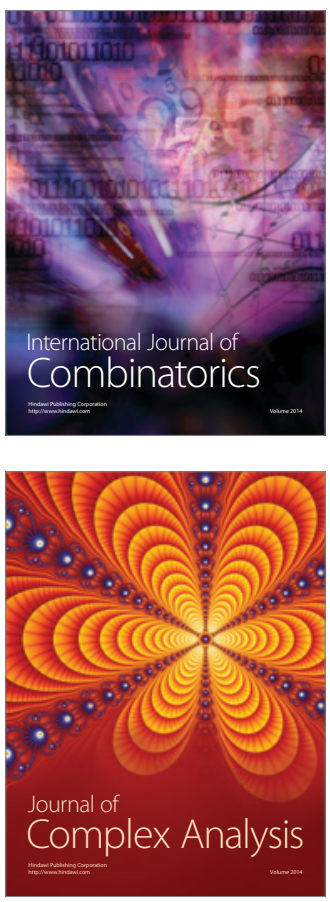

International Journal of

Mathematics and

Mathematical

Sciences
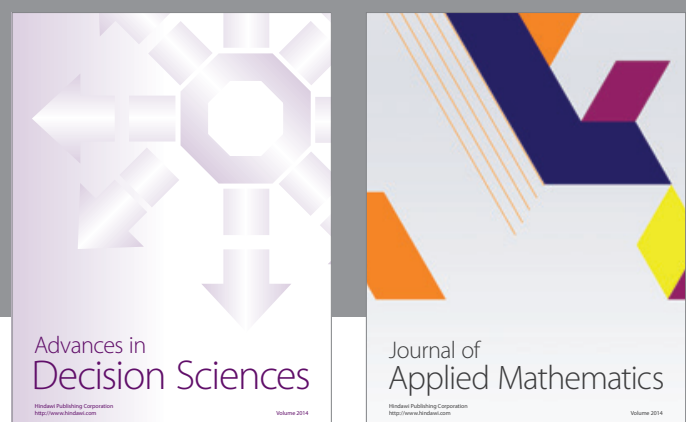

Journal of

Applied Mathematics
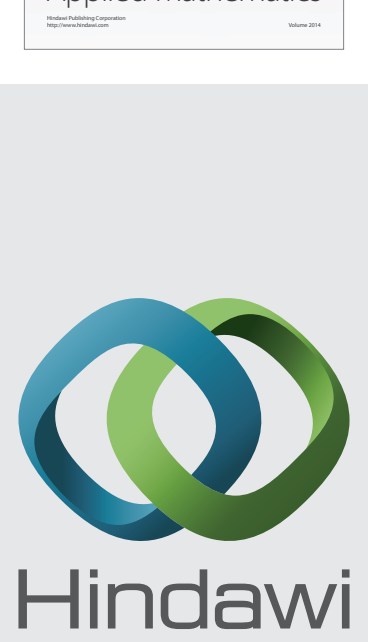

Submit your manuscripts at http://www.hindawi.com
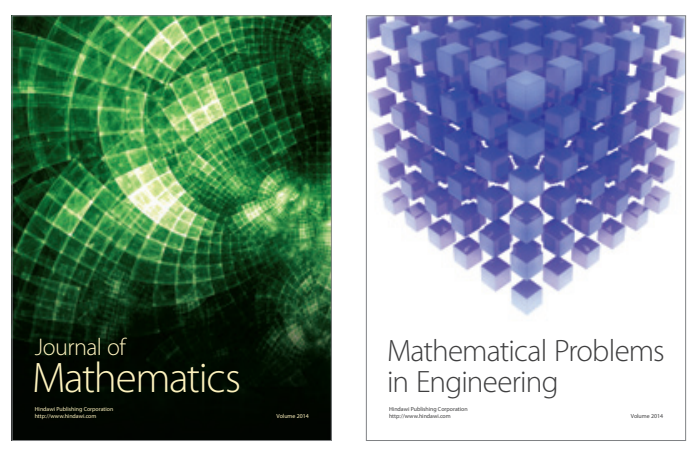

Mathematical Problems in Engineering
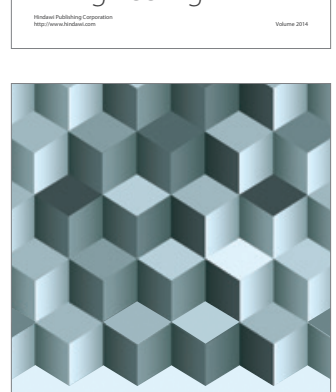

Journal of

Function Spaces
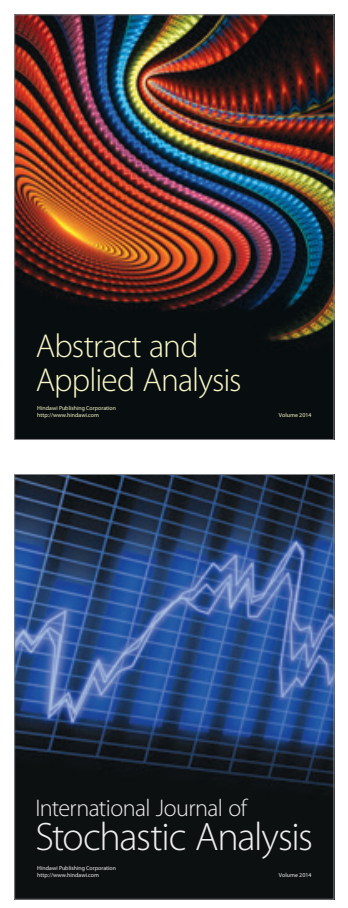

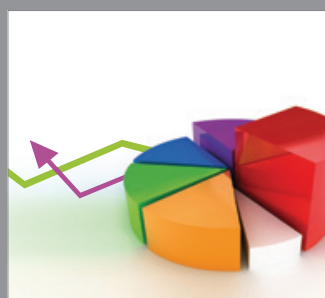

ournal of

Probability and Statistics

Promensencen
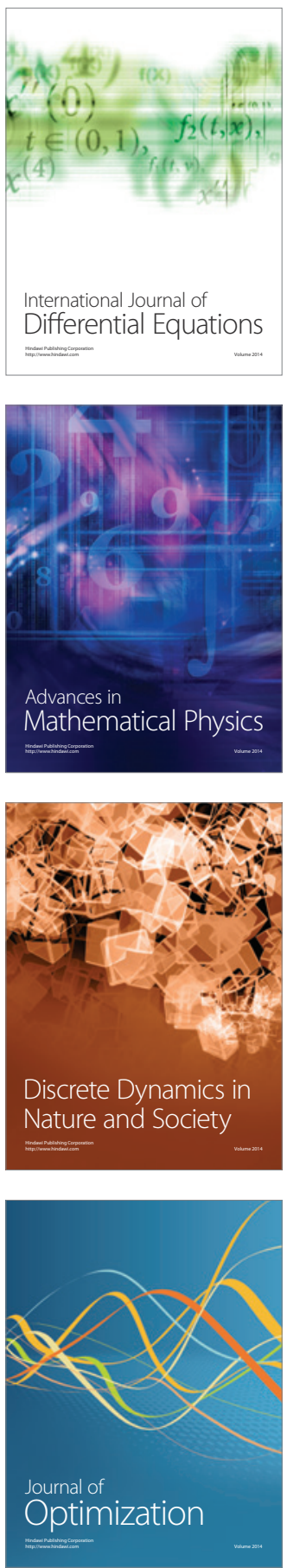